\title{
Suzaku-WAM soft gamma-ray all-sky monitor by the earth occultation technique
}

\author{
Chie Kira ${ }^{a}$, Yasushi Fukazawa ${ }^{a}$, Tetsuya Asano ${ }^{a}$, Hiromitsu Takahashi ${ }^{a}$, Takuya \\ Takahashi $^{a}$, Takeshi Uehara ${ }^{a}$, Yoshitaka Hanabata ${ }^{a}$, Kazutaka Yamaoka $^{b}$, Makoto \\ Tashiro $^{c}$, Yukikatsu Terada ${ }^{c}$, Masanori Ohno ${ }^{d}$ \\ ${ }^{a}$ Hiroshima University, Japan \\ ${ }^{b}$ Aoyama Gakuin University, Japan \\ ${ }^{c}$ Saitama University, Japan \\ ${ }^{d}$ ISAS/JAXA, Japan \\ E-mail: kira@hep01.hepl.hiroshima-u.ac.jp
}

Suzaku Wide-band All-sky Monitor (WAM), which is the BGO Active Shield surrounding the Hard X-ray Detector (HXD) on-board Suzaku, has detected many gamma-ray bursts, soft gammaray repeaters, and solar flares. In addition, thanks to its large field of view ( $2 \pi$ str), WAM can monitor bright soft gamma-ray sources with the earth occultation technique, as CGRO/BATSE. We have constructed the analysis system of the WAM earth occultation, calibrated the obtained spectra and flux by the Crab nebula, and found that the response uncertainty is around $20 \%$. Therefore, WAM is an important all-sky monitor in the unique $50 \mathrm{keV}-1 \mathrm{MeV}$ band against RXTE/ASM (1 - $10 \mathrm{keV})$ and Swift/BAT $(10-100 \mathrm{keV})$, and enables us to monitor objects in wide energy band from $1 \mathrm{keV}$ to $700 \mathrm{keV}$ by utilizing these three instruments, as well as $I N$ TEGRAL. This is a great advantage to observe the hard tail of black hole binaries and search non-thermal components and $511 \mathrm{keV}$ lines associated with the jet ejection. Here, we introduce preliminary results on Galactic black hole binaries. The long-term light curve of Cyg X-1 is successfully obtained around $100-600 \mathrm{keV}$ with WAM. Together with light curves of RXTE/ASM and Swift/BAT, we can clearly see the different behavior between them. Noticeably, the bright phase in 2006 September is coincident with a detection at $0.1-1 \mathrm{TeV}$ energies by MAGIC telescope. This event was also reported by RXTE/ASM, Swift/BAT and INTEGRAL/IBIS and SPI. In the average spectrum up to $700 \mathrm{keV}$ of Cyg X-1, high energy cut-off is clearly seen. Furthermore, we obtained a monthly light curve and spectrum of GRS 1915+105, GX 339-4 and average spectra of some X-ray binaries. Many gamma-ray flares from black hole binaries are expected to be found by Fermi, and simultaneous X-ray monitoring with RXTE, Swift, INTEGRAL, and Suzaku is very important to constrain the emission mechanism.

7th INTEGRAL Workshop

Copenhagen, Denmark

September, 8-11 2008 


\section{Introduction}

It is believed that most of black holes are accompanied by jets. So as to investigate the mechanism of the jet emission, it is important to study non-thermal components of jet origin in soft gamma-ray region. However, still the soft gamma-ray emission from black holes is not well understood. In the low/hard state, is there a non-thermal component or $511 \mathrm{keV}$ line associated with the jet emission beyond the normal thermal-Compton components? In the high/soft state, where is the cut-off for the power-law component? How about the very high state? In order to give answers to these questions, it is essential to study the spectral changes at the state transition. Hence, it is very important to monitor black holes continuously, especially in the energy band between $100 \mathrm{keV}$ and $1 \mathrm{MeV}$, and catch the state transition. Here we introduce the Suzaku-WAM soft gamma-ray all-sky monitor by the earth occultation technique, and show preliminary results on the black hole binaries.

\section{WAM ( Wide-band All-sky Monitor )}

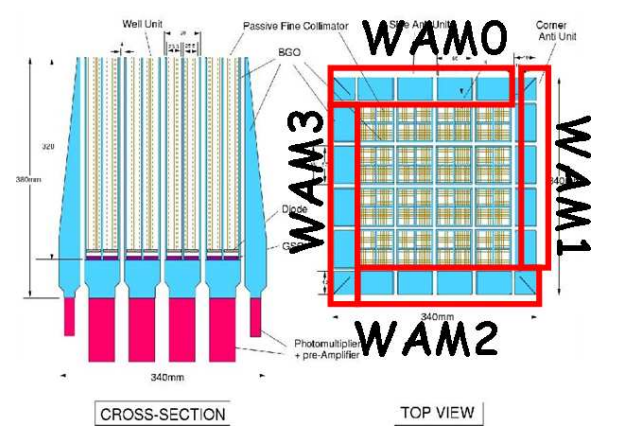

Figure 1: Overview of Suzaku-WAM

Suzaku-WAM (Wide-band All-sky Monitor) is the active shield of the HXD (Hard X-ray Detector) constructed from thick BGO scintillators and photomultipliers (Fig.1]; [1]). It surrounds four sides of HXD, and each side is called WAM0, WAM1, WAM2, and WAM3. WAM is also a detector with very large field of view ( $2 \pi$ str), wide energy range $(50-5000$ $\mathrm{keV})$, and large effective area $\left(800 \mathrm{~cm}^{2}\right)$ to observe many gamma-ray bursts, soft gamma-ray repeaters, and solar fhres. In addition, thanks to its large field of view, WAM can monitor bright soft gamma-ray sources of at least $50 \mathrm{mCrab}$ with the earth occultation technique, as CGRO/BATSE [2]. Current all-sky monitors are RXTE/ASM (1.5 - $12 \mathrm{keV})$ and Swift/BAT (15 - $150 \mathrm{keV})$. WAM is unique against such monitors for its high energy band, and enables us to monitor objects in wide energy band from $1 \mathrm{keV}$ to $700 \mathrm{keV}$, by utilizing these three instruments. This is a great advantage to observe the hard tail of black hole binaries and search non-thermal components or $511 \mathrm{keV}$ line associated with the jet ejection.

\section{Analysis Procedure}

\subsection{Calculate Occultation Time}

At first, we select the observations in which the incident angle of a target object to each WAM detector is smaller than 50 degrees, and calculate the time of occultation from target position and satellite orbit. There are about 15 occultations in one day, and each occultation is recognized by two steps of count rate at the begin and end of the occultation in the light curve. 


\subsection{Extract Occultation Light Curve}

We analyze Suzaku-WAM TRN-data. TRN-data contain light curves of 55 energy channels with a time resolution of $1 \mathrm{sec}$. Here, we treat the light curves of $1-27 \mathrm{ch}$ in $50-1000 \mathrm{keV}$, and extract the light curve before and after $500 \mathrm{sec}$ for each occultation in each energy channel.

Since the earth occultation time calculated above is obtained without considering the atmospheric absorption, we must correct it before summing over the occultation light curves. The effect of atmosphere depends on the object track on the earth, seen from the satellite. Therefore, we obtained the relation between the observed time of occultation and the duration of occultation for the Crab and Cygnus X-1. Using the obtained relation, we correct the time from the duration of predicted occultation. We used only the data whose occultation duration time is longer than 1200 sec. We eliminated the occultation light curves containing SAA, background high-rate period, and calibration mode, and summing over the occultation light curves in one observation, one month, or three years for each energy channel to increase the statistics. WAM2 data are not in use because of its large calibration uncertainty.

\subsection{Quantify Occultation Step Count Rate}

We fit an occultation light curve in each energy channel by an appropriate function to quantify the step count rate (Fig.2). Here, occultation light curves at the begin and end are simultaneously fitted with the same step count rate. We derive the spectrum of the object from the step count rates in each energy channel.

\subsection{Calculate the Response Function}

We calculate the response function from the incident direction of the object to the WAM. The response is created for one observation, during which incident direction is the same. In the case of the occultation

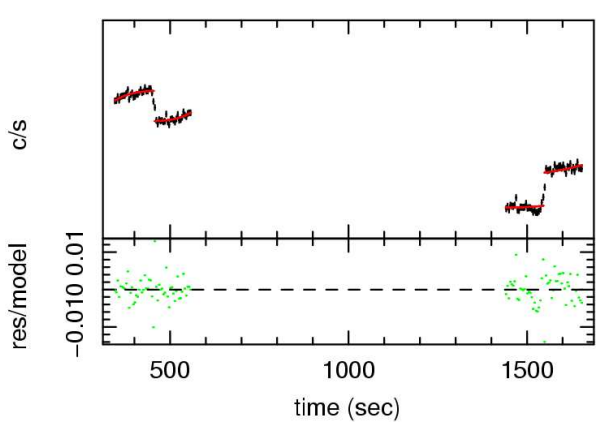

Figure 2: Example of fitting earth occultation step of the Crab (One day integration) light curves summing over one month or three years, we averaged the responses too, weighted by number of occultations in each observation.

\subsection{Make the Ancillary Response File}

Because of the absorption by structure of satellite, at this time, WAM response function might overestimate the flux especially below $100 \mathrm{keV}$, and the uncertainty depends on the incident angle. We modeled the difference between the observed count rate and model count rate predicted by the response as function of its incident angle by using the Crab, and correct response files. This correction reduces the uncertainty of response files from $40 \%$ to $20 \%$. 


\section{Current Detection Uncertainty}

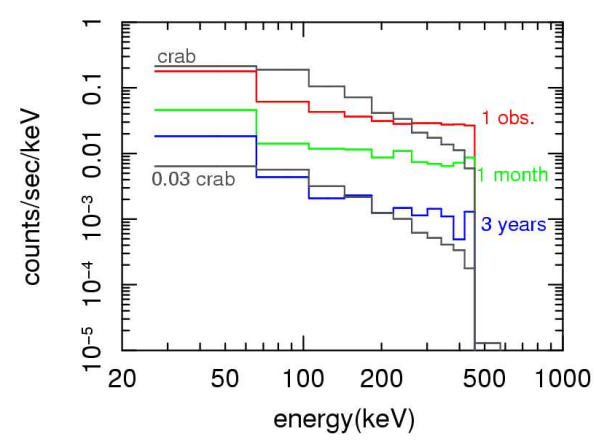

Figure 3: Current detection limit

Detection of celestial objects is mostly limited by the photon statistics of background events. We analyzed the step count rate of 11 dark objects for WAM in the same method as above, and derived the systematic uncertainty of detection by estimating the variance of the obtained step count rates for these dark objects (Fig]).

Three-year integration gives systematic errors of $0.03 \mathrm{Crab}$ at $100 \mathrm{keV}$. However, the analysis procedure is not yet polished well, and therefore the uncertainty is currently somewhat worse than the above.

\section{Calibration by the Crab}

The incident direction varies with the satellite attitude, and the WAM response function strongly depends on the incident direction due to the surrounding satellite body and other instruments. Therefore, we first calibrated the response function by the Crab for various incident directions. As shown in Fig. $\bigoplus$, in three-year integration, Crab can be detected up to $300 \mathrm{keV}$ in one observation integration, during which the satellite attitude is the same.

We fitted the spectrum of each observation by power-law model with a photon index fixed to 2.1, and obtained the flux in $100-500 \mathrm{keV}$. We confirmed that the flux does not depend on the elapsed time from launch within $20 \%$ accuracy. On the other hand, it is significant that the flux depends on the incident angle to each WAM detector above 60 degrees. Therefore, we used the observation data below 50 degrees.

In three-year integration, the Crab can be detected up to $800 \mathrm{keV}$ (Fig.Đ). The spectrum is well fitted by power-law model with a photon index of 2.1 and flux of $1.5 \times 10^{-8} \mathrm{erg} / \mathrm{sec} / \mathrm{cm}^{2}(100-500 \mathrm{keV})$. These are roughly consistent with the INTEGRAL results [3].

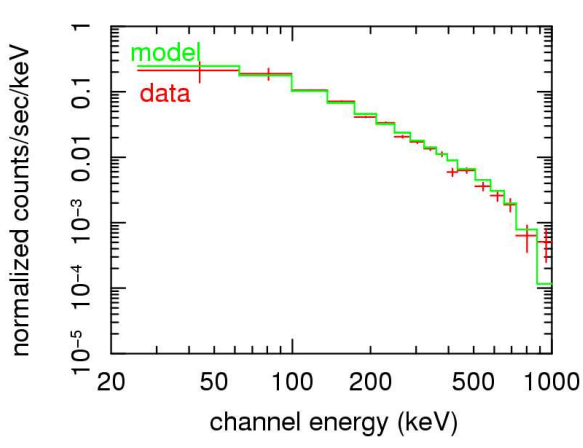

Figure 4: Averaged spectrum of the Crab in three-year integration

\section{Preliminary Results}

\subsection{Long-term Light Curve of Cyg X-1}

The long-term light curve in one observation integration of Cyg X-1 is successfully obtained in $100-600 \mathrm{keV}$ with WAM as shown in Fig.5. Together with light curves of RXTE/ASM and Swift/BAT, we can clearly see the different behavior between them. 
Noticeably, the bright phase in 2006 September $(M J D \simeq 54000)$ is coincident with a high energy gamma-ray detection at $0.1-1 \mathrm{TeV}$ energies by MAGIC telescope [ 4 . This event was also reported by RXTE/ASM, Swift/BAT and INTEGRAL/IBIS and SPI [5]. The next noticeable is a dark phase above $10 \mathrm{keV}$ against a bright flare below $10 \mathrm{keV}$ in 2006 April - May (MJD $\simeq 53830)$. Although there was no report that Cyg X-1 made a state transition in this period, it shows a transition to a sort of intermediate state.

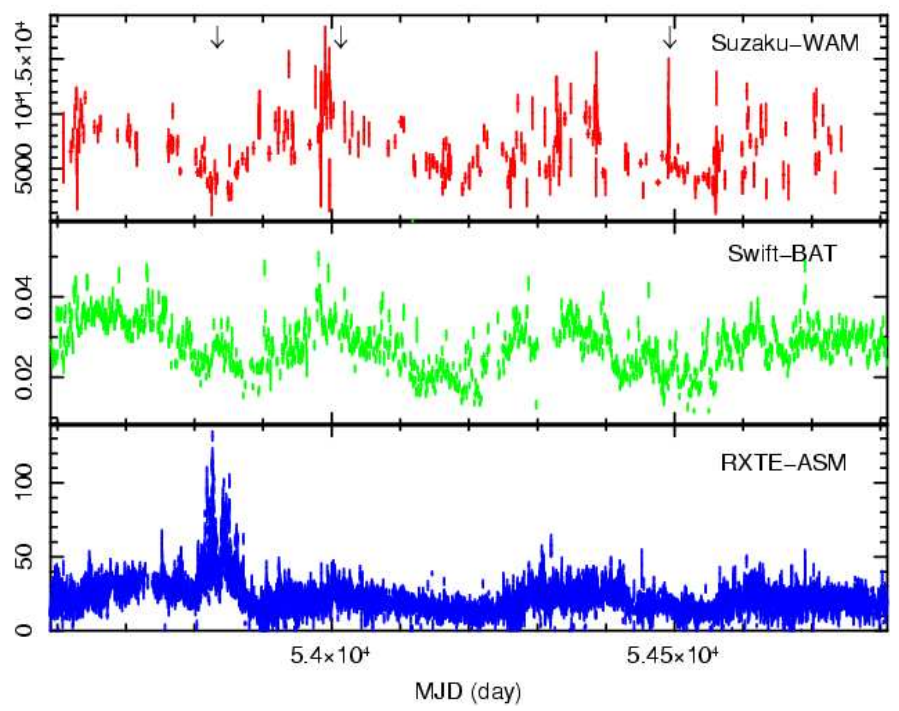

Figure 5: Long-term light curve of Cyg X-1. Arrows represent epoch A, B and C in Fig]

\subsection{Spectra of Cyg X-1 at Various Epochs}

Fig. 6 shows the average spectrum in three-year integration of Cyg X-1, compared with the spectrum of the Crab with a photon index of 2.1. High energy cutoff is clearly seen. It is well fitted by cut-off power-law model with a photon index of 1.5 and a cut-off at 200 $\mathrm{keV}$.

We also obtained wide-band X-ray spectra of ASM/BAT/WAM at 3 epochs. We converted ASM and BAT count rate in the light curve to the spectra, considering the response function.

Fig.7 top-left panel is a spectrum in 2008 January (MJD $\simeq 54480$; epoch A) during which the flux is at

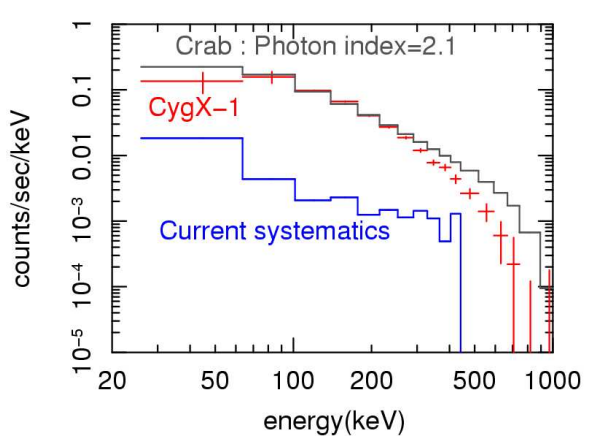

Figure 6: Averaged spectrum of Cyg X-1 in three-year integration the average level and Cyg X-1 is in typical low/hard state. This figure shows a fitting result of the compPS model. The other three panels in Fig.7 show the spectral energy distributions $(v F v)$ at 3 epochs, where the green line represents the model for Crab. Epoch B is a period during which Cyg X-1 seems like a intermediate state in 2006 April (MJD $\simeq 53830$ ), and epoch $\mathrm{C}$ is a period of TeV gamma-ray flare in 2006 September (MJD $\simeq 54000$ ).

Compared with epoch A or averaged spectrum (Fig.6), the spectrum at epoch B (intermediatestate-like period) seems softer. Although Malzac et al. (2008) reported that the spectral properties in the TeV fare period with INTEGRAL data are typical of those observed in the low/hard state, the one-month integrated WAM spectrum at epoch $\mathrm{C}(\mathrm{TeV}$ flare) seems harder. 

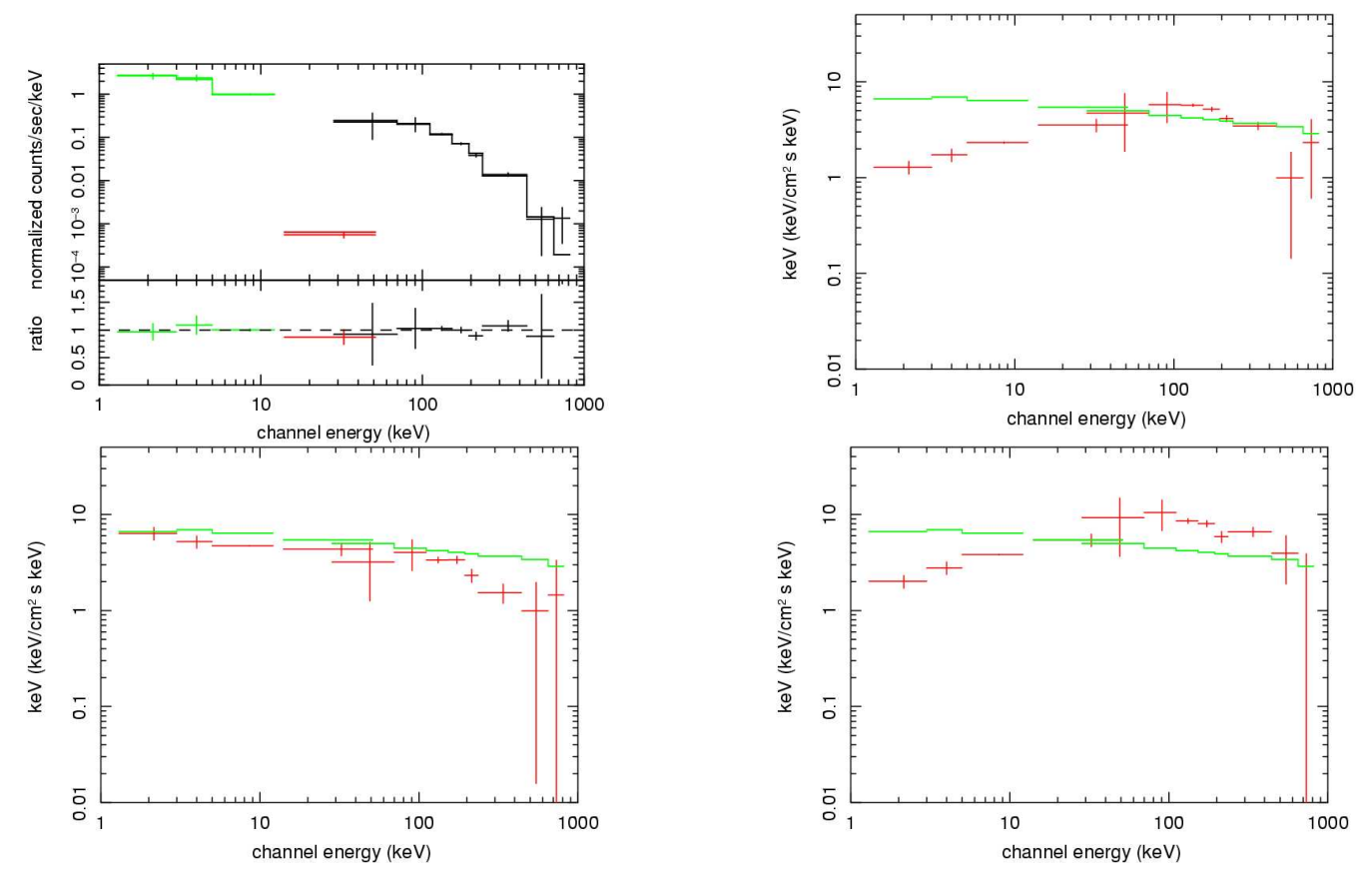

Figure 7: Top-left shows a joint fitting of spectra with RXTE/ASM and Swift/BAT at the epoch A (2008 Jan. 15 - 2008 Feb. 09). The other panels are spectral energy distributions of Cyg X-1 at three epochs of A, B (2006 Mar. 27 - 2006 Apr. 23), and C (2006 Sep. 20 - 2006 Oct. 17). The green represents the model for Crab.

\subsection{GRS 1915+105}

We obtained a monthly light curve and spectrum of GRS 1915+105.

Fig.8 shows a monthly light curve of GRS 1915+105. While the count rate of Swift is almost the half of Cyg X-1, the count rate between $100-400 \mathrm{keV}$ is much lower for GRS 1915+105, although the WAM detection is significant. During the normal hard state (state C [6]) before $\mathrm{MJD} \simeq 54250$, GRS $1915+105$ was continuously detected. After entering the soft state (state A), the detection becomes difficult.

Fig.9 shows the average spectrum of GRS $1915+105$ in threeyear integration. It is detected up

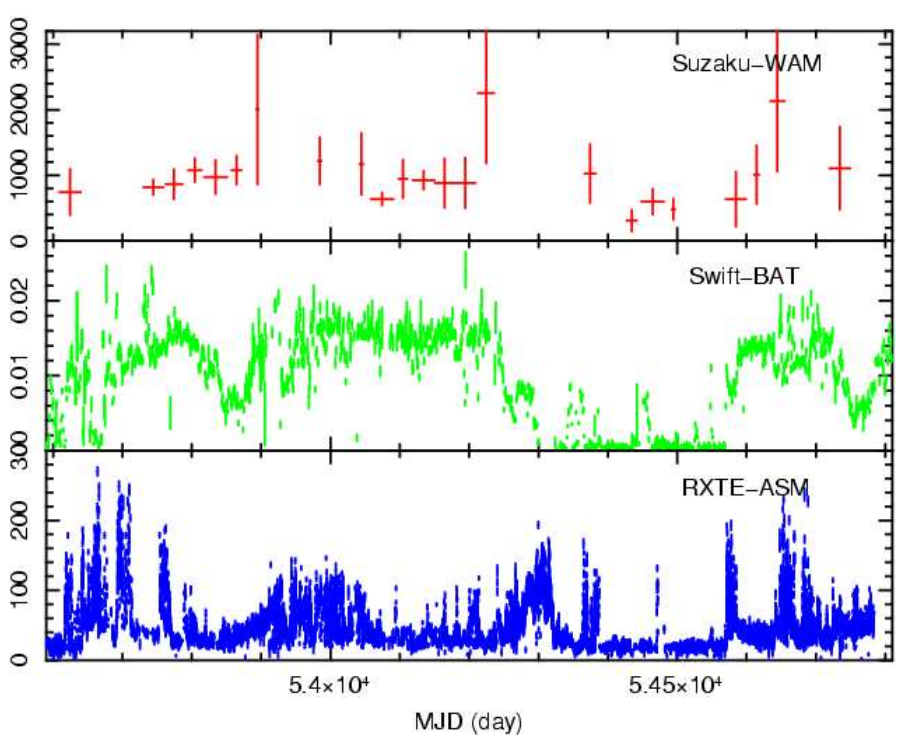

Figure 8: Monthly WAM light curve of GRS 1915+105 together with the light curves of Swift/BAT and RXTE/ASM to $400 \mathrm{keV}$, and the photon index is around 3. It can be seen that the spectrum consists of soft and 
hard components. Fig.10 shows the spectrum at the state A. In the spectrum, the thermal component is dominant in the lower energy of $<10 \mathrm{keV}$, and there is a hard tail above $10 \mathrm{keV}$. This is a possibility the nonthermal Comptonization as reported by [7]

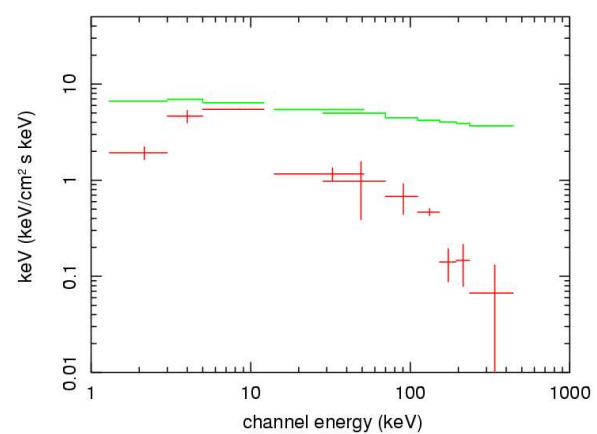

Figure 9: Averaged spectrum of GRS 1915+105 in three-year integration. The red crosses show data for GRS 1915+105 and the green lines model for Crab.

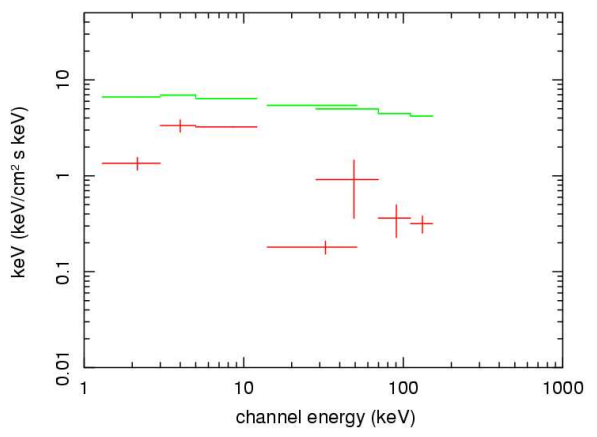

Figure 10: Spectrum of GRS $1915+105$ at the soft state. The red crosses show data for GRS $1915+105$ and the green lines model for Crab.

\subsection{GX 339-4}

GX 339-4 can also be monitored with one-month interval. In the light curve with WAM shown in Fig.11, the outburst around 2006 Dec. to 2007 Mar. is prominent.

It is well known that GX 339-4 shows a direct correlation and hysteresis between X-ray luminosity and spectral state [8]. The sub-state transition from the hard-intermediate state to the softintermediate state have been observed in past activities [9][10]. Similarly, in 2006 - 2007 outburst, such a transition was suggested with INTEGRAL observation [11][12] which was started from 2007 January 30th but INTE-

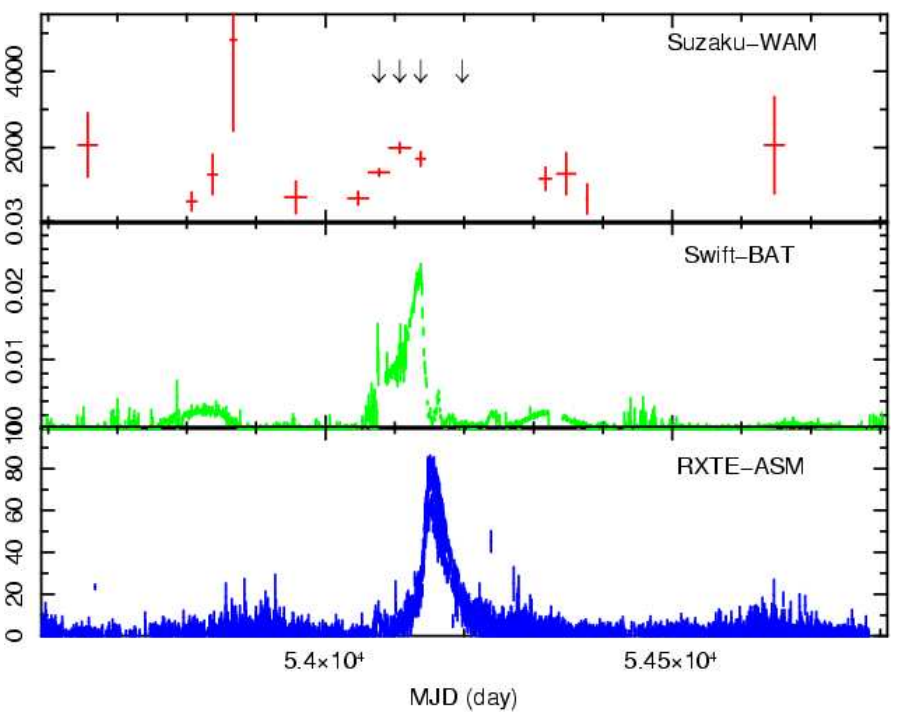

Figure 11: Monthly light curve of GX 339-4. The red crosses show data for GX 339-4 and the green lines model for Crab. Arrows represent epoch A, B, C and D in Fig.12 GRAL did not observe the main transition.

WAM could catch the rising phase of the burst and monitor the state transition. Compared with Swift/BAT and RXTE/ASM, the light curve at higher energy band seems to rise earlier, and this behavior indicates a transition to softer state. Fig.12 is the change of ASM/BAT/WAM spectral energy distributions during the burst $(\mathrm{MJD} \simeq 54120)$. Fig. 12 top-left panel shows $v F v$ spectrum at 
the early phase in the outburst (epoch A; 2006 Nov. 26 - 2006 Dec. 22). It is similar to that of the low/hard state of Cyg X-1 indicating that GX 339-4 brightened with the low/hard-like spectral shape. After that it got soften, and at last made transition to the soft state at epoch D (2007 Mar. 28 - 2007 Apr. 19).
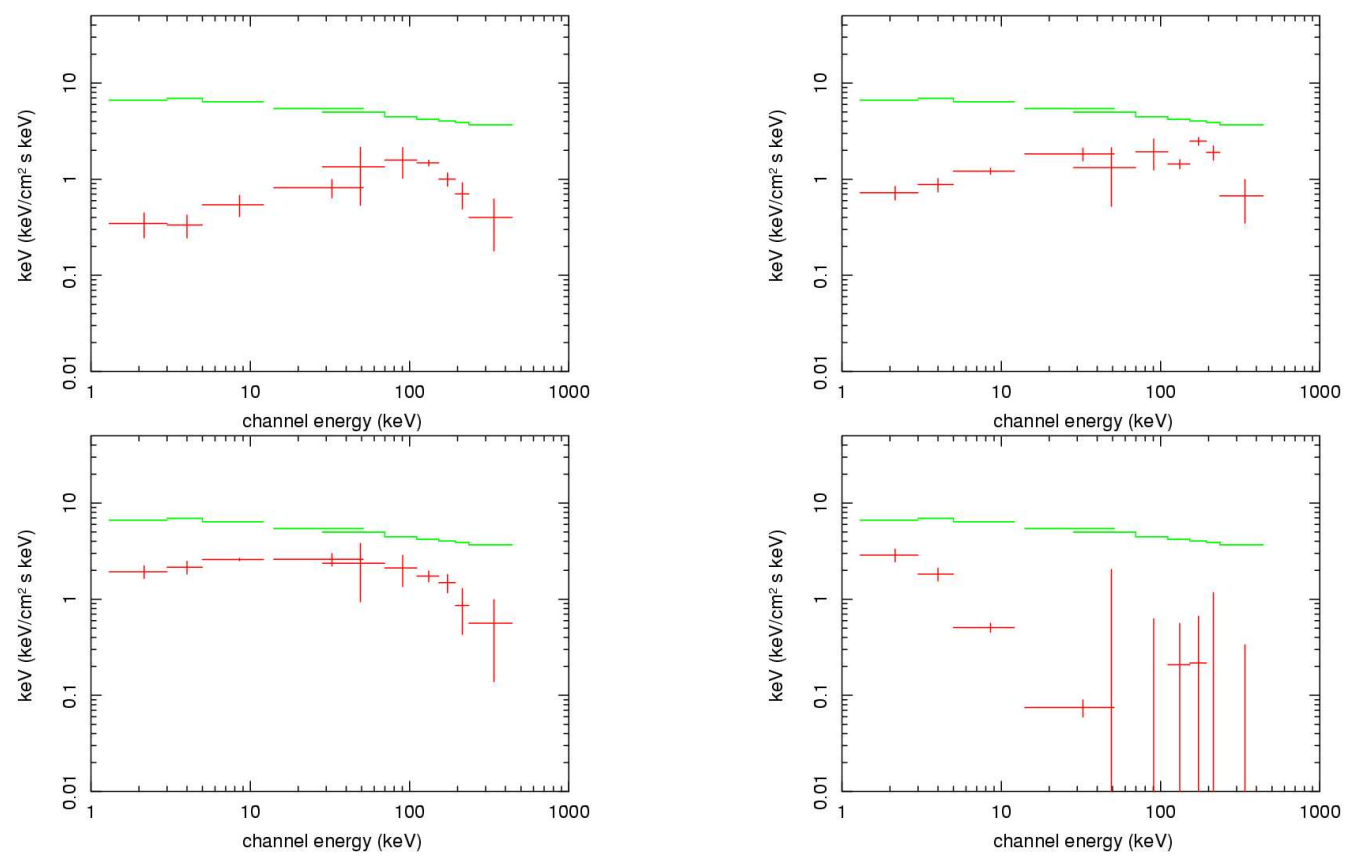

Figure 12: Changes of spectral energy distributions of GX 339-4 at flare around MJD 54120. Top-left shows the spectrum at epoch A (2006 Nov. 26 - 2006 Dec. 23), top-right B(2006 Dec. 23 - 2007 Jan.21), bottom-left C(2007 Jan. 22 - 2007 Feb. 11), and bottom-right D(2007 Mar. 28 - 2007 Apr. 19). The red crosses shows data for GX 339-4 and the green lines model for Crab.

\section{References}

[1] Yamaoka et al. 2009, PASJ, 61, S35

[2] Ling et al. 2000, ApJS 127, 79

[3] Sizun et al. 2004, astro-ph $/ 0 \overline{4} 0 \overline{6} 65 \overline{2} ;$; Proc. of the 5th INTEGRAL Workshop

[4] Albert et al. 2007, ApJ, 665, L51

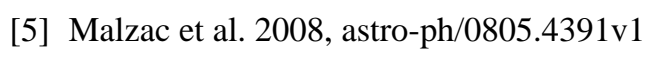

[6] Belloni et al. 2000, A\&A, 355, 271

[7] Zdziarski et al. 2001, ApJ, 554, L45

[8] Zdziarski and Gierlinski 2004, Progress of Theoretical Physics, 155, 99

[9] Fender et al. 2004, MNRAS, 355, 1105

[10] Belloni et al. 2004, ATel, 236

[11] Del Santo et al. 2008, MNRAS, 390, 227

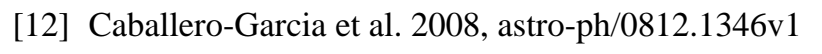

